\title{
An interior proximal cutting hyperplane method for equilibrium problems
}

Pham Ngoc Anh and Jong Kyu Kim ${ }^{*}$

* Correspondence:

jongkyuk@kyungnam.ac.kr

Department of Mathematics

Education, Kyungnam University,

Masan, Kyungnam, 631-701, Korea

\begin{abstract}
We propose a new method for solving equilibrium problems on polyhedra, where the underlying function is continuous and satisfies a pseudomonone assumption which is called an interior proximal cutting hyperplane method. The method is based on the special interior proximal function which replaces the usual quadratic function. This leads to an interior proximal algorithm. The algorithm can be viewed as combining the cutting hyperplane method and the special interior proximal function. Finally some preliminary computational results are given.

AMS Mathematics Subject Classification 2000: 65K10; 90 C25.
\end{abstract}

Keywords: Equilibrium problems, pseudomonotone, interior proximal function, cutting hyperplane method

\section{Introduction}

Equilibrium problems appear frequently in many practical problems arising, for instance, physics, engineering, game theory, transportation, economics and network (see $[1,2])$. They become an attractive field for many researchers both theory and applications (see [3-8]). These problems are models whose formulation includes optimization, variational inequalities, (vector) optimization problems, fixed point problems, saddle point problems, Nash equilibria and complementarity problems as particular cases (see $[1,5,9]$ ). In this article, we consider the equilibrium problems (shortly EP $(f, C))$ :

Find $x^{*} \in C$ such that $f\left(x^{*}, y\right) \geq 0$ for all $y \in C$,

where $C$ is a polyhedral set on $\mathbb{R}^{n}$ defined by

$$
C:=\left\{x \in \mathbb{R}^{n} \mid A x \leq b\right\},
$$

$A$ is a $p \times n$ matrix, $b \in \mathbb{R}^{p}, f: C \times C \rightarrow \mathbb{R}$ is a bifunction such that $f(x, x)=0$ for every $x \in C$.

Throughout this article, we assume that:

(A.1) int $C=\{x \mid A x<b\}$ is nonempty.

(A.2) $f(x, \cdot)$ is convex on $C$ for all $x \in C$.

(A.3) $f$ is continuous on $C \times C$.

(A.4) The solution set $S$ of EP $(f, C)$ is nonempty. 
Theory of equilibrium problems has been studied extensively and intensively on the existence of solutions and generalization to many abstract ways. However, methods for solving $\operatorname{EP}(f, C)$ still restrict and have not satisfy the need of applications. There are popular approaches for solving $\operatorname{EP}(f, C)$ to our knowledge. The first approach is based on the gap function (see [10]), the second way is to use the proximal point method [7] and the third one is the auxiliary subproblem principle [8].

In [3,4], Anh proposed interior proximal methods for solving monotone equilibrium problems when $C$ is a polyhedral convex set. The method is based on a special interior proximal function which replaces the usual quadratic function. The method has also been studied to variational inequalities by many authors (see $[11,12]$ ). This leads to an interior proximal-type algorithm, which can be viewed as combining an Armijo-type line search technique and the special interior proximal function. The only assumption required is that $f$ is monotone on $C$.

In this article, we propose an algorithm for solving $\mathrm{EP}(f, C)$, by making no assumptions on the problem other than continuity and pseudomonotonicity of the bifunction $f$. Recently, Anh and Kuno [13] introduced a new method for solving multivalued variational inequalities on a closed convex set, where the underlying function is upper semicontinuous and generalized monotone. We extend the cutting hyperplane method to $\operatorname{EP}(f, C)$. First, we construct an appropriate hyperplane which separates the current iterative point from the solution set. Next we combine this technique with Armijo-type line search technique to obtain a convergent algorithm for pseudomonotone equilibrium problems. Then the next iteration is obtained as the projection of the current iteration onto the intersection of the feasible set with the halfspace containing the solution set.

The article is organized as follows. In Section 2, we give formal definitions of our target $\operatorname{EP}(f, C)$ and the pseudomonotonicity of $f$. We then combined an idea often used for multivalued variational inequalities to $\operatorname{EP}(f, C)$ and interior proximal technique to develop an iterative algorithm. Section 3 is devoted to the proof of its global convergence to a solution of $\operatorname{EP}(f, C)$. In the last section, we apply the algorithm for the Nash-Cournot oligopolistic market equilibrium model. The numerical results are implemented to verify our development.

\section{The interior proximal cutting hyperplane algorithm}

We list some well known definitions and the projection under the Euclidean norm which will be required in our following analysis.

Definition 2.1 Let $C$ be a closed convex subset of $\mathbb{R}^{n}$, we denote the projection on $C$ by $\operatorname{Pr}(\cdot)$, i.e,

$$
\operatorname{Pr}_{C}(x)=\arg \min \{\|y-x\| \mid y \in C\} \quad \forall x \in \mathbb{R}^{n} .
$$

Then the bifunction $f: C \times C \rightarrow \mathbb{R} \cup\{+\infty\}$ is said to be

(i) monotone on $C$ if for each $x, y \in C$,

$$
f(x, y)+f(y, x) \leq 0 ;
$$

(ii) pseudomonotone on $C$ if for each $x, y \in C$,

$$
f(x, y) \geq 0 \text { implies } f(y, x) \leq 0 .
$$


It is observe that $(i) \Rightarrow(i i)$. But the converse is not true. There are some examples in [14].

Classical variational inequality problems (shortly VIP) are to find a vector $x^{*} \in C$ such that

$$
\left\langle F\left(x^{*}\right), y-x^{*}\right\rangle \geq 0 \quad \forall y \in C,
$$

where $C \subseteq \mathbb{R}^{n}$ is a nonempty closed convex subset of $\mathbb{R}^{n}$ and $F$ is a continuous mapping from $C$ into $\mathbb{R}^{n}$. Then they can be alternatively formulated as finding the zero point of the operator $T(x)=F(x)+N_{C}(x)$ where

$$
N_{C}(x)= \begin{cases}\{y \in C \mid\langle y, z-x\rangle \leq 0, \forall z \in C\} & \text { if } x \in C, \\ \emptyset & \text { otherwise. }\end{cases}
$$

A well known method to solve this problem is the proximal point algorithm [2], which starting with any point $x^{0} \in C$ and $\lambda_{k} \geq \lambda>0$, iteratively updates $x^{k+1}$ conforming the following problem:

$$
0 \in \lambda_{k} T(x)+\nabla_{x} h\left(x, x^{k}\right)
$$

where

$$
h\left(x, x^{k}\right)=\frac{1}{2}\left\|x-x^{k}\right\|^{2} .
$$

Motivation for studying the algorithm of problem (2.1) could be found in $[11,15,16]$.

Auslender et al. [12] have proposed an interior proximal-type method for solving (VIP) on $C:=\mathbb{R}_{+}^{n}=\left\{x \in \mathbb{R}^{n} \mid x \geq 0\right\}$ through replacing function $h\left(x, x^{k}\right)$ by $d_{\varphi}\left(x, x^{k}\right)$ which is defined as

$$
d_{\phi}(x, y)=\sum_{i=1}^{n} y_{i}^{2} \phi\left(y_{i}^{-1} x_{i}\right)
$$

where

$$
\phi(t)= \begin{cases}\frac{v}{2}(t-1)^{2}+\mu(t-\log t-1) & \text { if } t>0, \\ +\infty & \text { otherwise }\end{cases}
$$

with $v>\mu>0$. The fundamental difference here is that the term $d_{\varphi}$ is used to force the iterations $\left\{x^{k+1}\right\}$ to stay in the interior of $\mathbb{R}_{+}^{n}$. This technique is extended by many authors to variational inequalities and equilibrium problems (see $[1,3]$ ).

Applying this idea to the equilibrium problem $\operatorname{EP}(f, C)$, we consider another interior proximal function defined by

$$
D(x, y)= \begin{cases}\frac{1}{2}\|x-y\|^{2}+\mu \sum_{i=1}^{p} l_{i}^{2}(y)\left(\frac{l_{i}(x)}{l_{i}(y)} \log \frac{l_{i}(x)}{l_{i}(y)}-\frac{l_{i}(x)}{l_{i}(y)}+1\right) & \text { if } x \in \text { int } C, \\ +\infty & \text { otherwise }\end{cases}
$$

with $\mu \in(0,1), a_{i}(i=1, \ldots, p)$ are the rows of the matrix $A$, and

$$
\begin{aligned}
l_{i}(x) & =b_{i}-\left\langle a_{i}, x\right\rangle, \\
l(x) & =\left(l_{1}(x), l_{2}(x), \ldots, l_{p}(x)\right)^{T} .
\end{aligned}
$$


We denote by $\nabla_{1} D(x, y)$ the gradient of $D(\cdot, y)$ at $x$ for every $y \in C$. It is easy to see that

$$
\nabla_{1} D(x, y)=x-y-\mu A^{T} X_{y} \log \frac{l(x)}{l(y)}
$$

where

$$
X_{y}:=\operatorname{diag}\left(l_{1}(y), \ldots, l_{p}(y)\right) \text { and } \log \frac{l(x)}{l(y)}:=\left(\log \frac{l_{1}(x)}{l_{1}(y)}, \ldots, \log \frac{l_{p}(x)}{l_{p}(y)}\right) .
$$

Then we consider the following regularized auxiliary problem (shortly RAP):

$$
\text { Find } x^{*} \in C \text { such that } f\left(x^{*}, y\right)+\frac{1}{c} D\left(y, x^{*}\right) \geq 0 \text { for all } y \in C \text {, }
$$

where $c>0$ is a regularization parameter.

The equivalence between $\operatorname{EP}(f, C)$ and $(R A P)$ is due to the following lemma (see [1]).

Lemma 2.2 Let $f: C \times C \rightarrow \mathbb{R} \cap\{+\infty\}$ be a bifunction and $x^{*} \in C$. Then $x^{*}$ is a solution to $\operatorname{EP}(f, C)$ if and only if $x *$ is a solution to (RAP).

Lemma 2.2 shows that the solution of the equilibrium problem $\operatorname{EP}(f, C)$ can be approximated by an iterative procedure $x^{k+1}=h\left(x^{k}\right), k=0,1, \ldots$, where $c>0, x^{0}$ is any starting point in $C$ and $h\left(x^{k}\right)$ is the unique solution of the strongly convex program:

$$
\min \left\{f\left(x^{k}, y\right)+\frac{1}{c} D\left(y, x^{k}\right) \mid y \in C\right\} .
$$

However, generally, the sequence $\left\{x^{k}\right\}$ does not converge to a solution of the equilibrium problems (see [2]).

Let $f$ be a mapping defined by

$$
f(x, y):=\sup \{\langle w, y-x\rangle \mid w \in F(x)\},
$$

where $F: C \rightarrow 2^{\mathbb{R}^{n}}$ is a multivalued mapping such that $F(x) \neq \emptyset$ for all $x \in C$. Then $\operatorname{EP}(f, C)$ can be formulated as the multivalued variational inequality problems (shortly MVIP):

Find $x^{*} \in C, w^{*} \in F\left(x^{*}\right)$ such that

$$
\left\langle w^{*}, x-x^{*}\right\rangle \geq 0 \quad \forall x \in C .
$$

In this case, it is known that solutions coincide with zeros of the following projected residual function

$$
T(x):=x-\operatorname{Pr}_{C}\left(x-w^{*}\right) .
$$

In other words, with $x^{0} \in C, w^{0} \in F\left(x^{0}\right)$, the point $\left(x^{0}, w^{0}\right)$ is a solution of (MVIP) if and only if $T\left(x^{0}\right)=0$, where $T\left(x^{0}\right)=x^{0}-\operatorname{Pr}_{C}\left(x^{0}-w^{0}\right)$ (see [16]). Applying this idea and interior proximal function technique $D(\cdot, \cdot)$ to the equilibrium problem $\operatorname{EP}(f, C)$, we obtain the solution scheme: Let $x^{k}$ be a current approximation to the solution of EP $(f, C)$. First, we compute $y^{k}=\arg \min \left\{f\left(x^{k}, y\right)+\beta D\left(y, x^{k}\right) \mid y \in C\right\}$ for some positive constant $\beta$. Next, we search the line segment between $x^{k}$ and $r\left(x^{k}\right)=x^{k}-y^{k}$ for a point $\left(\bar{w}^{k}, z^{k}\right)$ such that the hyperplane $\partial H_{k}=\left\{x \in \mathbb{R}^{n} \mid\left\langle\bar{w}^{k}, x-z^{k}\right\rangle=0\right\}$ strictly separates $x^{k}$ 
from the solution set $S$ of $\operatorname{EP}(f, C)$. To find such $\left(\bar{w}^{k}, z^{k}\right)$, we may use a computationally inexpensive Armijo-type procedure. Then we compute the next iterate $x^{k+1}$ by projecting $x^{k}$ onto the intersection of the feasible set $C$ with the halfspace $H_{k}=\left\{x \in \mathbb{R}^{n} \mid\left\langle\bar{w}^{k}, x-z^{k}\right\rangle \leq 0\right\}$.

Then, the algorithm is described as follows.

\section{Algorithm 2.3}

Step 0. Choose $\sigma>0, x^{0} \in C, 0<\sigma<\frac{\beta}{2}$, and $\gamma \in(0,1)$.

Step 1. Compute

$$
\gamma^{k}:=\arg \min \left\{f\left(x^{k}, y\right)+\beta D\left(y, x^{k}\right) \mid y \in C\right\}, r\left(x^{k}\right):=x^{k}-y^{k} .
$$

Find the smallest nonnegative number $m_{k}$ of $m$ such that

$$
f\left(x^{k}-\gamma^{m_{k}} r\left(x^{k}\right), y^{k}\right)+\sigma\left\|r\left(x^{k}\right)\right\|^{2} \leq 0 .
$$

Step 2. (Cutting hyperplane) Choose $\bar{w}^{k} \in \partial_{2} f\left(z^{k}, z^{k}\right)$, where $z^{k}:=x^{k}-\gamma^{m_{k}} r\left(x^{k}\right)$.

Set

$$
H_{k}:=\left\{x \in \mathbb{R}^{n} \mid\left\langle\bar{w}^{k}, x-z^{k}\right\rangle \leq 0\right\} .
$$

Find $x^{k+1}:=\operatorname{Pr}_{C \cap H_{k}}\left(x^{k}\right)$.

Step 3. Set $k:=k+1$, and go to Step 1 .

\section{Convergence of the algorithm}

In the next lemma, we justify the stopping criterion.

Lemma 3.1 If $r\left(x^{k}\right)=0$, then $x^{k}$ is a solution to equilibrium problem $\operatorname{EP}(f, C)$.

Proof. Since $y^{k}$ is the solution to problem (2.3) and an optimization result in convex programming (see [1]), we have

$$
0 \in \partial_{2} f\left(x^{k}, y^{k}\right)+\beta \nabla_{1} D\left(y^{k}, x^{k}\right)+N_{C}\left(y^{k}\right)
$$

where $N_{C}$ denotes the normal cone. From $y^{k} \in \operatorname{int} C$, it follows that $N_{C}\left(y^{k}\right)=\{0\}$. Hence

$$
\xi^{k}+\beta \nabla_{1} D\left(\gamma^{k}, x^{k}\right)=0,
$$

where $\xi^{k} \in \partial_{2} f\left(x^{k}, y^{k}\right)$. Replacing $y^{k}=x^{k}$ in this equality, we get

$$
\xi^{k}+\beta \nabla_{1} D\left(x^{k}, x^{k}\right)=0 .
$$

Since

$$
\nabla_{1} D(x, y)=x-y-\mu A^{T} X_{y} \log \frac{l(x)}{l(y)} \quad \forall x, y \in C,
$$

we have

$$
\nabla_{1} D\left(x^{k}, x^{k}\right)=0
$$


Thus $\xi^{k}=0$. Combining this with $f\left(x^{k}, x^{k}\right)=0$, we obtain

$$
f\left(x^{k}, y\right) \geq\left\langle\xi^{k}, y-\xi^{k}\right\rangle=0 \quad \forall y \in C .
$$

which means that $x^{k}$ is a solution to $\operatorname{EP}(f, C)$.

In Algorithm 2.3, we need to show the existence of the nonnegative integer $m_{k}$.

Lemma 3.2 For $\gamma \in(0,1), 0<\sigma<\frac{\beta}{2}$, if $r\left(x^{k}\right)>0$ then there exists the smallest nonnegative integer $m_{k}$ such that the inequality (2.4) holds.

Proof. Assume on the contrary, the inequality (2.4) is not satisfied for any nonnegative integer $i$, i.e.,

$$
f\left(x^{k}-\gamma^{i} r\left(x^{k}\right), \gamma^{k}\right)+\sigma\left\|r\left(x^{k}\right)\right\|^{2}>0 .
$$

Letting $i \rightarrow \infty$, from the continuity of $f$ we have

$$
f\left(x^{k}, \gamma^{k}\right)+\sigma\left\|r\left(x^{k}\right)\right\| \geq 0 .
$$

Otherwise, for each $t>0$ we have $1-\frac{1}{t} \leq \log t$. We obtain after multiplication by $\frac{l_{i}\left(y^{k}\right)}{l_{i}\left(x^{k}\right)}>0$ for each $i=1, \ldots, p$,

$$
\frac{l_{i}\left(y^{k}\right)}{l_{i}\left(x^{k}\right)}-1 \leq \frac{l_{i}\left(y^{k}\right)}{l_{i}\left(x^{k}\right)} \log \frac{l_{i}\left(y^{k}\right)}{l_{i}\left(x^{k}\right)} .
$$

Then,

$$
\begin{aligned}
D\left(y^{k}, x^{k}\right) & =\frac{1}{2}\left\|x^{k}-y^{k}\right\|^{2}+\mu \sum_{i=1}^{n} l_{i}^{2}\left(x^{k}\right)\left(\frac{l_{i}\left(y^{k}\right)}{l_{i}\left(x^{k}\right)} \log \frac{l_{i}\left(y^{k}\right)}{l_{i}\left(x^{k}\right)}-\frac{l_{i}\left(y^{k}\right)}{l_{i}\left(x^{k}\right)}+1\right) \\
& \geq \frac{1}{2}\left\|r\left(x^{k}\right)\right\|^{2} .
\end{aligned}
$$

Since $y^{k}$ is the solution to the strongly convex program (2.4), we have

$$
f\left(x^{k}, y\right)+\beta D\left(y, x^{k}\right) \geq f\left(x^{k}, y^{k}\right)+\beta D\left(y^{k}, x^{k}\right) \quad \forall y \in C .
$$

Substituting $y=x^{k} \in C$ and using assumptions $f\left(x^{k}, x^{k}\right)=0, D\left(x^{k}, x^{k}\right)=0$, we get

$$
f\left(x^{k}, y^{k}\right)+\beta D\left(y^{k}, x^{k}\right) \leq 0 .
$$

Combining (3.3) with (3.4), we obtain

$$
f\left(x^{k}, y^{k}\right)+\frac{\beta}{2}\left\|r\left(x^{k}\right)\right\|^{2} \leq 0 .
$$

Then, inequalities (3.2) and (3.5) imply that

$$
-\sigma\left\|r\left(x^{k}\right)\right\|^{2} \leq f\left(x^{k}, \gamma^{k}\right) \leq-\frac{\beta}{2}\left\|r\left(x^{k}\right)\right\|^{2} .
$$

Hence it must be either $r\left(x^{k}\right)=0$ or $\sigma \geq \frac{\beta}{2}$. The first case contradicts to $r\left(x^{k}\right) \neq 0$, while the second one contradicts to the fact $\sigma<\frac{\beta}{2}$.

The following results perform some property of the cutting hyperplane $H_{k}$. 
Lemma 3.3 Let $\left\{x^{k}\right\}$ be the sequence generated by Algorithm 2.3. Then the following hold:

(i) $x^{k} \notin H_{k}, S \subseteq C \cap H_{k}$.

(ii) $x^{k+1}=\operatorname{pr}_{C \cap H_{k}}\left(\bar{y}^{k}\right)$, where $\bar{y}^{k}=\operatorname{Pr}_{H_{k}}\left(x^{k}\right)$.

Proof. (i) Since $\bar{w}^{k} \in \partial_{2} f\left(z^{k}, z^{k}\right), \gamma^{k} \in C, f\left(z^{k}, z^{k}\right)=0$, and $z^{k}=x^{k}-\gamma^{m_{k}} r\left(x^{k}\right)$, we have

$$
\begin{aligned}
f\left(z^{k}, \gamma^{k}\right) & \geq\left\langle\bar{w}^{k}, \gamma^{k}-z^{k}\right\rangle \\
& =-\left(1+\gamma^{m_{k}}\right)\left\langle\bar{w}^{k}, r\left(x^{k}\right)\right\rangle .
\end{aligned}
$$

Combining this with (2.4), we obtain that

$$
\left(1+\gamma^{m_{k}}\right)\left\langle\bar{w}^{k}, r\left(x^{k}\right)\right\rangle \geq \sigma\left\|r\left(x^{k}\right)\right\|^{2} .
$$

Hence

$$
\begin{aligned}
\left\langle\bar{w}^{k}, x^{k}-z^{k}\right\rangle & =\gamma^{m_{k}}\left\langle\bar{w}^{k}, r\left(x^{k}\right)\right\rangle \\
& \geq \frac{\sigma \gamma^{m_{k}}}{1+\gamma^{m_{k}}}\left\|r\left(x^{k}\right)\right\|^{2} \\
& >0 .
\end{aligned}
$$

This implies $x^{k} \notin H_{k}$.

Since $f$ is assumed to be pseudomonotone on $C, z^{k} \in C$ and $x^{*} \in S$,

$$
f\left(x^{*}, z^{k}\right) \geq 0 \Rightarrow f\left(z^{k}, x^{*}\right) \leq 0 .
$$

Combining this with $\bar{w}^{k} \in \partial_{2} f\left(z^{k}, z^{k}\right)$, we get

$$
\begin{aligned}
\left\langle\bar{w}^{k}, x^{*}-z^{k}\right\rangle & \leq f\left(z^{k}, x^{*}\right)-f\left(z^{k}, z^{k}\right) \\
& \leq 0
\end{aligned}
$$

Thus, $x^{*} \in H_{k}$.

(ii) We know that

$$
H=\left\{x \in \mathbb{R}^{n} \mid\left\langle w, x-x^{0}\right\rangle \leq 0\right\}, \operatorname{Pr}_{H}(y)=y-\frac{\left\langle w, y-x^{0}\right\rangle}{\|w\|^{2}} w .
$$

Hence,

$$
\begin{aligned}
\bar{y}^{k} & =\operatorname{Pr}_{H_{k}}\left(x^{k}\right) \\
& =x^{k}-\frac{\left\langle\bar{w}^{k}, x^{k}-z^{k}\right\rangle}{\left\|\bar{w}^{k}\right\|^{2}} \bar{w}^{k} \\
& =x^{k}-\frac{\gamma^{m_{k}}\left\langle\bar{w}^{k}, r\left(x^{k}\right)\right\rangle}{\left\|\bar{w}^{k}\right\|^{2}} \bar{w}^{k} .
\end{aligned}
$$

Otherwise, for every $y \in C \cap H_{k}$ there exists $\lambda \in(0,1)$ such that

$$
\hat{x}=\lambda x^{k}+(1-\lambda) y \in C \cap \partial H_{k}
$$


where $\partial H_{k}=\left\{x \in \mathbb{R}^{n} \mid\left\langle\bar{w}^{k}, x-z^{k}\right\rangle=0\right\}$, because $x^{k} \in C$ but $x^{k} \notin H_{k}$.

$$
\begin{aligned}
\left\|y-\bar{y}^{k}\right\|^{2} & \geq(1-\lambda)^{2}\left\|y-\bar{y}^{k}\right\|^{2} \\
& =\left\|\hat{x}-\lambda x^{k}-(1-\lambda) \bar{y}^{k}\right\|^{2} \\
& =\left\|\left(\hat{x}-\bar{y}^{k}\right)-\lambda\left(x^{k}-\bar{y}^{k}\right)\right\|^{2} \\
& =\left\|\hat{x}-\bar{y}^{k}\right\|^{2}+\lambda^{2}\left\|x^{k}-\bar{y}^{k}\right\|^{2}-2 \lambda\left\langle\hat{x}-\bar{y}^{k}, x^{k}-\bar{y}^{k}\right\rangle \\
& =\left\|\hat{x}-\bar{y}^{k}\right\|^{2}+\lambda^{2}\left\|x^{k}-\bar{y}^{k}\right\|^{2} \\
& \geq\left\|\hat{x}-\bar{y}^{k}\right\|^{2},
\end{aligned}
$$

because $\bar{\gamma}^{k}=\operatorname{Pr}_{H_{k}}\left(x^{k}\right)$. Also we have

$$
\begin{aligned}
\left\|\hat{x}-x^{k}\right\|^{2} & =\left\|\hat{x}-\bar{y}^{k}+\bar{y}^{k}-x^{k}\right\|^{2} \\
& =\left\|\hat{x}-\bar{y}^{k}\right\|^{2}-2\left\langle\hat{x}-\bar{y}^{k}, x^{k}-\bar{y}^{k}\right\rangle+\left\|\bar{y}^{k}-x^{k}\right\|^{2} \\
& =\left\|\hat{x}-\bar{y}^{k}\right\|^{2}+\left\|\bar{y}^{k}-x^{k}\right\|^{2} .
\end{aligned}
$$

Since $x^{k+1}=\operatorname{Pr}_{C \cap H_{k}}\left(x^{k}\right)$, using the Pythagorean theorem we can reduce that

$$
\begin{aligned}
\left\|\hat{x}-\bar{y}^{k}\right\|^{2} & =\left\|\hat{x}-x^{k}\right\|^{2}-\left\|\bar{y}^{k}-x^{k}\right\|^{2} \\
& \geq\left\|x^{k+1}-x^{k}\right\|^{2}-\left\|\bar{y}^{k}-x^{k}\right\|^{2} \\
& =\left\|x^{k+1}-\bar{y}^{k}\right\|^{2} .
\end{aligned}
$$

From (3.7) and (3.8), we have

$$
\left\|x^{k+1}-\bar{y}^{k}\right\| \leq\left\|y-\bar{y}^{k}\right\| \quad \forall y \in C \cap H_{k}
$$

which implies

$$
x^{k+1}=\operatorname{Pr}_{\mathrm{C} \cap H_{k}}\left(\bar{\gamma}^{k}\right) .
$$

In order to prove the convergence of Algorithm 2.3, we give the following key property of the sequence $\left\{x^{k}\right\}$ generated by the algorithm.

Lemma 3.4 The sequence $\left\{x^{k}\right\}$ generated by Algorithm 2.3 satisfies the following inequality.

$$
\left\|x^{k+1}-x^{*}\right\|^{2} \leq\left\|x^{k}-x^{*}\right\|^{2}-\left\|x^{k+1}-\gamma^{k}\right\|^{2}-\left(\frac{\gamma^{m_{k}} \sigma}{\left\|\bar{w}^{k}\right\|\left(1+\gamma^{m_{k}}\right)}\right)^{2}\left\|r\left(x^{k}\right)\right\|^{4} .
$$

Proof. Since $x^{k+1}=\operatorname{Pr}_{C \cap H_{k}}\left(\gamma^{k}\right)$, we have

$$
\left\langle y^{k}-x^{k+1}, z-x^{k+1}\right\rangle \leq 0 \quad \forall z \in C \cap H_{k} .
$$


Ant and Kim Journal of Inequalities and Applications 2012, 2012:99

Page 9 of 14

Substituting $z=x^{*} \in C \cap H_{k}$, then we have

$$
\left\langle y^{k}-x^{k+1}, x^{*}-x^{k+1}\right\rangle \leq 0 \Leftrightarrow\left\langle y^{k}-x^{k+1}, x^{*}-y^{k}+y^{k}-x^{k+1}\right\rangle \leq 0,
$$

which implies

$$
\left\|x^{k+1}-y^{k}\right\|^{2} \leq\left\langle x^{k+1}-\gamma^{k}, x^{*}-y^{k}\right\rangle .
$$

Hence,

$$
\begin{aligned}
\left\|x^{k+1}-x^{*}\right\|^{2} & =\left\|x^{k+1}-y^{k}+y^{k}-x^{*}\right\|^{2} \\
& =\left\|x^{k+1}-y^{k}\right\|^{2}+\left\|y^{k}-x^{*}\right\|^{2}+2\left\langle x^{k+1}-y^{k}, y^{k}-x^{*}\right\rangle \\
& \leq\left\langle x^{*}-y^{k}, x^{k+1}-y^{k}\right\rangle+\left\|y^{k}-x^{*}\right\|^{2}+2\left\langle x^{k+1}-y^{k}, y^{k}-x^{*}\right\rangle \\
& =\left\|y^{k}-x^{*}\right\|^{2}+\left\langle x^{k+1}-y^{k}, y^{k}-x^{*}\right\rangle \\
& =\left\|y^{k}-x^{*}\right\|^{2}-\left\|x^{k+1}-y^{k}\right\|^{2} .
\end{aligned}
$$

Since $z^{k}=x^{k}-\gamma^{m_{k}} r\left(x^{k}\right)$ and

$$
y^{k}-\operatorname{Pr}_{H_{k}}\left(x^{k}\right)=x^{k}-\frac{\left\langle\bar{w}^{k}, x^{k}-z^{k}\right\rangle}{\left\|\bar{w}^{k}\right\|^{2}} \bar{w}^{k},
$$

we have

$$
\begin{aligned}
\left\|y^{k}-x^{*}\right\|^{2} & \\
= & \left\|x^{k}-x^{*}\right\|^{2}+\frac{\left\langle\bar{w}^{k}, x^{k}-z^{k}\right\rangle^{2}}{\left\|\bar{w}^{k}\right\|^{4}}\left\|\bar{w}^{k}\right\|^{2}-\frac{2\left\langle\bar{w}^{k}, x^{k}-z^{k}\right\rangle}{\left\|\bar{w}^{k}\right\|^{2}}\left\langle\bar{w}^{k}, x^{k}-x^{*}\right\rangle \\
= & \left\|x^{k}-x^{*}\right\|^{2}+\left(\frac{\gamma^{m_{k}}\left\langle\bar{w}^{k}, r\left(x^{k}\right)\right\rangle}{\left\|\bar{w}^{k}\right\|}\right)^{2}-\frac{2 \gamma^{m_{k}}\left\langle\bar{w}^{k}, r\left(x^{k}\right)\right\rangle}{\left\|\bar{w}^{k}\right\|^{2}}\left\langle\bar{w}^{k}, x^{k}-x^{*}\right\rangle \\
= & \left\|x^{k}-x^{*}\right\|^{2}-\left(\frac{\gamma^{m_{k}}\left\langle\bar{w}^{k}, r\left(x^{k}\right)\right\rangle}{\left\|\bar{w}^{k}\right\|}\right)^{2} \\
& -2\left[\frac{\gamma^{m_{k}}\left\langle\bar{w}^{k}, r\left(x^{k}\right)\right\rangle}{\left\|\bar{w}^{k}\right\|^{2}}\left\langle\bar{w}^{k}, x^{k}-x^{*}\right\rangle-\left(\frac{\gamma^{m_{k}}\left\langle\bar{w}^{k}, r\left(x^{k}\right)\right\rangle}{\left\|\bar{w}^{k}\right\|}\right)^{2}\right] \\
= & \left\|x^{k}-x^{*}\right\|^{2}-\left(\frac{\gamma^{m_{k}}\left\langle\bar{w}^{k}, r\left(x^{k}\right)\right\rangle}{\left\|\bar{w}^{k}\right\|}\right)^{2} \\
& -\frac{2 \gamma^{m_{k}}\left\langle\bar{w}^{k}, r\left(x^{k}\right)\right\rangle}{\left\|\bar{w}^{k}\right\|^{2}}\left[\left\langle\bar{w}^{k}, x^{k}-x^{*}\right\rangle-\gamma^{m_{k}}\left\langle\bar{w}^{k}, r\left(x^{k}\right)\right\rangle\right] \\
= & \left\|x^{k}-x^{*}\right\|^{2}-\left(\frac{\gamma^{m_{k}}\left\langle\bar{w}^{k}, r\left(x^{k}\right)\right\rangle}{\left\|\bar{w}^{k}\right\|}\right)^{2} \\
& -\frac{2 \gamma^{m_{k}}\left\langle\bar{w}^{k}, r\left(x^{k}\right)\right\rangle}{\left\|\bar{w}^{k}\right\|^{2}}\left\langle\bar{w}^{k}, x^{k}-x^{*}-\gamma^{m_{k}} r\left(x^{k}\right)\right\rangle \\
= & \left\|x^{k}-x^{*}\right\|^{2}-\left(\frac{\gamma^{m_{k}}\left\langle\bar{w}^{k}, r\left(x^{k}\right)\right\rangle}{\left\|\bar{w}^{k}\right\|}\right)^{2}-\frac{2 \gamma^{m_{k}}\left\langle\bar{w}^{k}, r\left(x^{k}\right)\right\rangle}{\left\|\bar{w}^{k}\right\|^{2}}\left\langle\bar{w}^{k}, z^{k}-x^{*}\right\rangle .
\end{aligned}
$$


From (3.6), it follows that

$$
\left\langle\bar{w}^{k}, r\left(x^{k}\right)\right\rangle \geq \frac{\sigma}{1+\gamma^{m_{k}}}\left\|r\left(x^{k}\right)\right\|^{2} .
$$

Thus, (3.11) reduces to

$$
\begin{aligned}
\left\|y^{k}-x^{*}\right\|^{2} & \leq\left\|x^{k}-x^{*}\right\|^{2}-\left(\frac{\gamma^{m_{k}}\left\langle\bar{w}^{k}, r\left(x^{k}\right)\right\rangle}{\left\|\bar{w}^{k}\right\|}\right)^{2} \\
& \leq\left\|x^{k}-x^{*}\right\|^{2}-\left(\frac{\gamma^{m_{k}} \sigma}{\left\|\bar{w}^{k}\right\|\left(1+\gamma^{m_{k}}\right)}\right)^{2}\left\|r\left(x^{k}\right)\right\|^{4} .
\end{aligned}
$$

Combining (3.10) and (3.12), we obtain the inequality (3.9)

Theorem 3.5 Suppose that Assumptions A.1-A.4 hold, the mapping $\partial_{2} f\left(\cdot, z^{k}\right)$ is uniformly bounded by $M>0$, and $f$ is pseudomonone on $C$. Then the sequence $\left\{x^{k}\right\}$ generated by Algorithm 2.3 converges to a solution of $\mathrm{EP}(f, C)$.

Proof. The inequality (3.9) implies that the sequence $\left\{\left\|x^{k}-x^{*}\right\|\right\}$ is nonincreasing and hence convergent. Consequently, the sequence $\left\{x^{k}\right\}$ is bounded.

Since the mapping $\partial_{2} f\left(\cdot, z^{h}\right)$ is uniformly bounded by $M>0$, i.e.,

$$
\left\|w^{k}\right\| \leq M \quad \forall k=1, \ldots
$$

This, together with (3.9), implies

$$
\left\|x^{k+1}-x^{*}\right\|^{2} \leq\left\|x^{k}-x^{*}\right\|^{2}-\left\|x^{k+1}-\gamma^{k}\right\|^{2}-\left(\frac{\gamma^{m_{k}} \sigma}{M\left(1+\gamma^{m_{k}}\right)}\right)^{2}\left\|r\left(x^{4}\right)\right\|^{4} .
$$

Since $\left\{\left\|x^{k}-x^{*}\right\|\right\}$ converges to zero, it is easy to see that

$$
\lim _{k \rightarrow \infty} \gamma^{m_{k}}\left\|r\left(x^{k}\right)\right\|=0 .
$$

The cases remaining to consider are the following.

Case 1. $\limsup _{k \rightarrow \infty} \gamma^{m_{k}}>0$. This case must follow that $\liminf _{k \rightarrow \infty}\left\|r\left(x^{k}\right)\right\|=0$. Since $\left\{x^{k}\right\}$ is bounded, there exists $\bar{x}$ which is an accumulation point of $\left\{x^{k}\right\}$. In other words, a subsequence $\left\{x^{k_{i}}\right\}$ converges to some $\bar{x}$ such that $r(\bar{x})=0$, as $i \rightarrow \infty$. Then we see from Lemma 3.3 that $\bar{x} \in S$, and besides we can take $x^{*}=\bar{x}$, in particular in (3.13). Thus $\left\{\left\|x^{k}-\bar{x}\right\|\right\}$ is a convergent sequence. Since $\bar{x}$ is an accumulation point of $\left\{x^{k}\right\}$, the sequence $\left\{\left\|x^{k}-x^{*}\right\|\right\}$ converges to zero, i.e., $\left\{x^{k}\right\}$ converges to $\bar{x} \in S$.

Case 2. $\lim _{k \rightarrow \infty} \gamma^{m_{k}}=0$. Since $m_{k}$ is the smallest nonnegative integer, $m_{k}-1$ does not satisfy (2.4). Hence, we have

$$
f\left(x^{k}-\gamma^{m_{k-1}} r\left(x^{k}\right), \gamma^{k}\right)>-\sigma\left\|r\left(x^{k}\right)\right\|^{2},
$$

and besides

$$
f\left(x^{k_{i}}-\gamma^{m_{k_{i}}-1} r\left(x^{k_{i}}\right), \gamma^{k_{i}}\right)>-\sigma\left\|r\left(x^{k_{i}}\right)\right\|^{2} .
$$


Passing onto the limit in (3.14), as $i \rightarrow \infty$, and using the continuity of $f$, we have

$$
f(\bar{x}, \bar{y}) \geq-\sigma\|\bar{x}-\bar{y}\|^{2} .
$$

From (3.5) we have

$$
f\left(x^{k_{i}}, y^{k_{i}}\right) \leq-\frac{\beta}{2} \| r\left(x^{k_{i}} \|^{2}\right.
$$

Since $f$ is continuous, passing onto the limit, as $i \rightarrow \infty$, we obtain

$$
f(\bar{x}, \bar{y}) \leq-\frac{\beta}{2}\|\bar{x}-\bar{y}\|^{2} .
$$

Combining this with (3.15), we have

$$
s \sigma\|\bar{x}-\bar{y}\|^{2} \geq \frac{\beta}{2}\|\bar{x}-\bar{y}\|^{2},
$$

which implies $r(\bar{x}):=\|\bar{x}-\bar{y}\|=0$ or $\sigma \geq \frac{\beta}{2}$. The second case contradicts to the fact $0<\sigma<\frac{\beta}{2}$ and hence $r(\bar{x})=0, \bar{x} \in S$. Letting $x^{*}=\bar{x}$ and repeating the previous arguments, we conclude that the whole sequence $\left\{x^{k}\right\}$ converges to $\bar{x} \in S$.

\section{Numerical results}

We applied the algorithm to solve a problem of production competition under the Nash-Cournot oligopolistic market equilibrium model (see [1,2,17]). In this model, it is assumed that there are $n$-firms producing a common homogenous commodity and that the price $p_{i}$ of firm $i$ depends on the total quantity $\sigma_{x}=\sum_{i=1}^{n} x_{i}$ of the commodity.

Let $h_{i}\left(x_{i}\right)$ denote the cost of the firm $i$ when its production level is $x_{i}$. Suppose that the profit of firm $i$ is given by

$$
f_{i}\left(x_{1}, \ldots, x_{n}\right):=x_{i} p_{i}\left(\sigma_{x}\right)-h_{i}\left(x_{i}\right) \quad i=1, \ldots, n,
$$

where $h_{i}$ is the cost function of firm $i$ that is assumed to be dependent only on its production level.

Let $C \subset \mathbb{R}_{+}^{n}:=\left\{x \in \mathbb{R}^{n} \mid x \geq 0\right\}$ be closed convex which denotes the strategy set of firms. Each firm seeks to maximize its own profit by choosing the corresponding production level under the presumption that the production of the other firms are parametric input. In this context, a Nash equilibrium is a production pattern in which no firm can increase its profit by changing its controlled variables. Thus under this equilibrium concept, each firm determines its best response given other firms' actions. Mathematically, a point $x^{*}=\left(x_{1}^{*}, \ldots, x_{n}^{*}\right) \in C$ is said to be a Nash equilibrium point if

$$
f_{i}\left(x_{1}^{*}, \ldots, x_{i-1}^{*}, y_{i}, x_{i+1}^{*}, \ldots, x_{n}^{*}\right) \leq f_{i}\left(x_{1}^{*}, \ldots, x_{n}^{*}\right) \quad \forall y \in C .
$$

When $h_{i}$ is affine, this market problem can be formulated as a special Nash equilibrium problem in the $n$-person nonco-operative game theory.

Set

$$
\phi(x, y):=-\sum_{i=1}^{n} f_{i}\left(x_{1}, \ldots, x_{i-1}, y_{i}, x_{i+1}, \ldots, x_{n}\right)
$$


and

$$
\begin{aligned}
f(x, y): & =\phi(x, y)-\phi(x, x) \\
& =\sum_{i=1}^{n}\left(h_{i}\left(y_{i}\right)-h_{i}\left(x_{i}\right)-y_{i} p\left(y_{i}+\sum_{j \neq i} x_{j}\right)+x_{i} p\left(\sum_{i=1}^{n} x_{i}\right)\right) .
\end{aligned}
$$

Then it has been proved in [17] that the problem of finding an equilibrium point of this model can be formulated as $\operatorname{EP}(f, C)$ :

Find $x \in C$ such that $f\left(x^{*}, y\right) \geq 0, \quad$ for all $y \in C$.

Proposition 4.1 [2]A point $x^{*}$ is an equilibrium point for the oligopolistic market problem if and only if it is a solution to $\operatorname{EP}(f, C)$, where

$$
\begin{aligned}
& f(x, y):=\left\langle H(x)-p\left(\sigma_{x}\right) e-p^{\prime}\left(\sigma_{x}\right) x, y-x\right\rangle \\
& H(x)=\left(h_{1}^{\prime}\left(x_{1}\right), \ldots, h_{n}^{\prime}\left(x_{n}\right)\right)^{T}, e=(1, \ldots, 1)^{T}, \sigma_{x}=\langle x, e\rangle .
\end{aligned}
$$

The following proposition gives some properties of the bifunction $f$.

Proposition 4.2 [2] Let $p: C \rightarrow \mathbb{R}_{+}$be convex, twice continuously differentiable, and nonincreasing and let the function $\mu_{\tau}: \mathbb{R}_{+} \rightarrow \mathbb{R}_{+}$, defined by $\mu_{\tau}\left(\sigma_{x}\right)=\sigma_{x} p\left(\sigma_{x}+\tau\right)$ be concave for every $\tau \geq 0$. Also, let the function $h_{i}: \mathbb{R}_{+} \rightarrow \mathbb{R}, i=1, \ldots, n$, be convex and twice continuously differentiable. Then, the cost bifunction

$$
f(x, y):=\left\langle H(x)-p\left(\sigma_{x}\right) e-p^{\prime}\left(\sigma_{x}\right) x, y-x\right\rangle
$$

is monotone on $C$.

We now applied the algorithm to the example with seven firms $(n=7)$ provided in $[9,17]$, where the cost and inverse demand functions have the form

$$
\begin{aligned}
& H(x):=\left(2 x_{1}+1,3 x_{2}+4,4 x_{3}+2,1.5 x_{4}+3,4 x_{5}+1, x_{6}-2,3 x_{7}+1\right) T, \\
& p(t):=\frac{2}{3 t} t \in(0,+\infty) .
\end{aligned}
$$

Then Propositions 4.1 and 4.2 show that the bifunction defined by (4.4) is monotone on $C \times C$ and therefore assumptions of our algorithm are satisfied.

In this example, we choose

$$
\begin{aligned}
n & =7 \\
\eta & =0.1 \\
\gamma & =2 \\
\beta & =5 \\
\mu & =0.5 \\
x^{0} & =(3,3,3,3,3,3,3)^{T} \\
C & =\left\{x \in \mathbb{R}^{n} \mid 13 \leq \sum_{i=1}^{n} x_{i} \leq 25,1 \leq x_{i} \leq 5(i=1, \ldots, n)\right\} .
\end{aligned}
$$

Note that in this case, at iteration $k$, we have

$$
\partial_{2} f\left(z^{k}, z^{k}\right)=\left\{H\left(z^{k}\right)-p\left(\sigma_{z^{k}}\right) e-p^{\prime}\left(\sigma_{z^{k}}\right) z^{k}\right\}
$$


Table 1 Approximate solutions for $k$

\begin{tabular}{cccccccc}
\hline Iter $(\boldsymbol{k})$ & $x_{1}^{k}$ & $x_{2}^{k}$ & $x_{3}^{k}$ & $x_{4}^{k}$ & $x_{5}^{k}$ & $x_{6}^{k}$ & $x_{7}^{k}$ \\
\hline 0 & 3 & 3 & 3 & 3 & 3 & 3 & 3 \\
1 & 2.0919 & 1.0015 & 1.0036 & 1.4664 & 1.0493 & 5.0019 & 1.3946 \\
2 & 2.0943 & 1.0020 & 0.9991 & 1.4648 & 1.0509 & 5.0019 & 1.3979 \\
3 & 2.0933 & 0.9993 & 0.9982 & 1.4608 & 1.0472 & 4.9992 & 1.3955 \\
4 & 2.0938 & 1.0000 & 1.0011 & 1.4617 & 1.0483 & 5.0003 & 1.3964 \\
5 & 2.0939 & 1.0002 & 1.0009 & 1.4618 & 1.0487 & 5.0007 & 1.3970 \\
6 & 2.0939 & 0.9998 & 0.9995 & 1.4607 & 1.0480 & 4.9998 & 1.3965 \\
7 & 2.0940 & 1.0000 & 1.0003 & 1.4610 & 1.0482 & 5.0001 & 1.3968 \\
\hline
\end{tabular}

where $p^{\prime}\left(\sigma_{z^{k}}\right)=-\frac{2}{3 \sigma_{z^{k}}^{2}}$. Lemma 3.1 shows that if $r\left(x^{k}\right)=0$, then $x^{k}$ is a solution to EP $(f, C)$. So we can say that $x^{k}$ is an $\epsilon$-solution to $\operatorname{EP}(f, C)$ if we have $\left\|r\left(x^{k}\right)\right\| \leq \epsilon$. The tolerance is taken by $\epsilon=10^{-6}$, we obtained the following Table 1 .

The approximate solution obtained after seven iterations is

$$
x^{7}=(2.0940,1.0000,1.0003,1.4610,1.0482,5.0001,1.3968)^{T} .
$$

\section{Acknowledgements}

This study was completed while the first author was staying at the Kyungnam University for the NRF Postdoctoral Fellowship for Foreign Researchers. And the second author was supported by the Kyungnam University Research Fund, 2011.

\section{Authors' contributions}

JKK conceived the study and participated in its design and coordination. JKK suggested many good ideas that are useful for achievement this paper and made the revision. PNA and JKK prepared the manuscript initially and performed all the steps of proof in this research. All authors read and approved the final manuscript.

\section{Competing interests}

The authors declare that they have no competing interests.

Received: 28 March 2011 Accepted: 23 April 2012 Published: 23 April 2012

\section{References}

1. Daniele, P, Giannessi, F, Maugeri, A: Equilibrium Problems and Variational Models. Kluwer, Academic Publisher (2003)

2. Konnov, IV: Combined Relaxation Methods for Variational Inequalities. Springer-Verlag, Berlin (2000)

3. Anh, PN: A logarithmic quadratic regularization method for solving pseudomonotone equilibrium problems. Acta Math Vietnam. 34, 183-200 (2009)

4. Anh, PN: An LQP regularization method for equilibrium problems on polyhedral. Vietnam J Math. 36, $209-228$ (2008)

5. Blum, E, Oettli, W: From optimization and variational inequality to equilibrium problems. Math Stud. 63, 127-149 (1994)

6. Mastroeni, G: Gap function for equilibrium problems. J Global Optim. 27, 411-426 (2004)

7. Moudafi, A: Proximal point algorithm extended to equilibrium problem. J Nat Geom. 15, 91-100 (1999)

8. Noor, MA: Auxiliary principle technique for equilibrium problems. J Optim Theory Appl. 122, 371-386 (2004)

9. Bigi, G, Castellani, M, Pappalardo, M: A new solution method for equilibrium problems. Optim Method Softw. 24, 895-911 (2009)

10. Mangasarian, OL, Solodov, MV: A linearly convergent derivative-free descent method for strongly monotone complementarity problem. Comput Optim Appl. 14, 5-16 (1999)

11. Anh, PN: An interior-quadratic proximal method for solving monotone generalized variational inequalities. East West $J$ Math. 10, 81-100 (2008)

12. Auslender, A, Teboulle, M, Bentiba, S: A logarithmic-quadratic proximal method for variational inequalities. J Comput Optim Appl. 12, 31-40 (1999)

13. Anh, PN, Kuno, T: A cutting hyperplane method for generalized monotone nonlips-chitzian multivalued variational inequalities. Modeling, Simulation and Optimization of Complex Processes. Springer Heidelberg (2012)

14. Schaible, S, Karamardian, S, Crouzeix, JP: Characterizations of generalized monotone maps. J Optim Theory Appl. 76 , 399-413 (1993)

15. Anh, PN, Muu, LD, Strodiot, JJ: Generalized projection method for non-Lipschitz multivalued monotone variational inequalities. Acta Math Vietnam. 34, 67-79 (2009)

16. Anh, PN, Muu, LD, Nguyen, VH, Strodiot, JJ: Using the Banach contraction principle to implement the proximal point method for multivalued monotone variational inequalities. J Optim Theory Appl. 124, 285-306 (2005) 
17. Murphy, FH, Sherali, HD, Soyster, AL: A mathematical programming approach for determining oligopolistic market equilibrium. Math Program. 24, 92-106 (1982)

doi:10.1186/1029-242X-2012-99

Cite this article as: Anh and Kim: An interior proximal cutting hyperplane method for equilibrium problems.

Journal of Inequalities and Applications 2012 2012:99.

Submit your manuscript to a SpringerOpen ${ }^{\odot}$ journal and benefit from:

- Convenient online submission

- Rigorous peer review

- Immediate publication on acceptance

- Open access: articles freely available online

- High visibility within the field

- Retaining the copyright to your article

Submit your next manuscript at $\boldsymbol{\nabla}$ springeropen.com 